OPEN ACCESS

Edited by:

Junhua Peng,

Center for Life Sci\&Tech of China National Seed Group Co. Ltd., China

Reviewed by:

Liang Chen,

University of Chinese Academy of Sciences (UCAS), China

Xiaoli Jin,

Zhejiang University, China

Guangxiao Yang,

Huazhong University of Science and Technology, China

*Correspondence: Leena Tripathi

l.tripathi@cgiar.org

${ }^{\dagger}$ Deceased

Specialty section: This article was submitted to

Plant Biotechnology, a section of the journal Frontiers in Plant Science

Received: 24 February 2017 Accepted: 30 May 2017

Published: 29 June 2017

Citation:

Muiruri KS, Britt A, Amugune NO, Nguu EK, Chan S and Tripathi L

(2017) Expressed Centromere Specific Histone 3 (CENH3) Variants in Cultivated Triploid and Wild Diploid

Bananas (Musa spp.).

Front. Plant Sci. 8:1034. doi: 10.3389/fpls.2017.01034

\section{Expressed Centromere Specific Histone 3 (CENH3) Variants in Cultivated Triploid and Wild Diploid Bananas (Musa spp.)}

\author{
Kariuki S. Muiruri ${ }^{1,2}$, Anne Britt ${ }^{3}$, Nelson O. Amugune ${ }^{2}$, Edward K. Nguu ${ }^{4}$, Simon Chan ${ }^{3+}$ \\ and Leena Tripathi ${ }^{1 *}$
}

\begin{abstract}
${ }^{1}$ International Institute of Tropical Agriculture, Nairobi, Kenya, ${ }^{2}$ School of Biological Sciences, University of Nairobi, Nairobi, Kenya, ${ }^{3}$ Department of Plant Biology, University of California, Davis, Davis, CA, United States, ${ }^{4}$ Department of Biochemistry, University of Nairobi, Nairobi, Kenya
\end{abstract}

Centromeres are specified by a centromere specific histone 3 (CENH3) protein, which exists in a complex environment, interacting with conserved proteins and rapidly evolving satellite DNA sequences. The interactions may become more challenging if multiple CENH3 versions are introduced into the zygote as this can affect post-zygotic mitosis and ultimately sexual reproduction. Here, we characterize CENH3 variant transcripts expressed in cultivated triploid and wild diploid progenitor bananas. We describe both splice- and allelic-[Single Nucleotide Polymorphisms (SNP)] variants and their effects on the predicted secondary structures of protein. Expressed CENH3 transcripts from six banana genotypes were characterized and clustered into three groups (MusaCENH-1A, MusaCENH-1B, and MusaCENH-2) based on similarity. The CENH3 groups differed with SNPs as well as presence of indels resulting from retained and/or skipped exons. The CENH3 transcripts from different banana genotypes were spliced in either 7/6, $5 / 4$ or $6 / 5$ exons/introns. The $7 / 6$ and the $5 / 4$ exon/intron structures were found in both diploids and triploids, however, $7 / 6$ was most predominant. The $6 / 5$ exon/introns structure was a result of failure of the $7 / 6$ to splice correctly. The various transcripts obtained were predicted to encode highly variable $\mathrm{N}$-terminal tails and a relatively conserved C-terminal histone fold domain (HFD). The SNPs were predicted in some cases to affect the secondary structure of protein by lengthening or shorting the affected domains. Sequencing of banana CENH3 transcripts predicts SNP variations that affect amino acid sequences and alternatively spliced transcripts. Most of these changes affect the $\mathrm{N}$-terminal tail of $\mathrm{CENH3}$.

Keywords: CENH3, splice variants, genotype, centromere, histones, banana

\section{INTRODUCTION}

Centromeres are assembly sites for the kinetochore, a protein complex that connects chromosomes to spindle fibers during meiosis and mitosis. The structure, size, and distribution of centromeres differ with species in spite of their common function (Talbert et al., 2004). Centromeres in both plants and animals often contain arrays of rapidly evolving tandemly repeated DNA sequences 
(Gent et al., 2011; Verdaasdonk and Bloom, 2011). The high rate of evolution in these repeats is remarkable given the fact that the function of centromeres is highly conserved. The role of the repeats is a subject of debate with the most common proposition being that they maintain the large heterochromatic domains associated with centromeres (Malik and Henikoff, 2009; Black and Cleveland, 2011). It is reported that CENH3 [aka Centromere Protein A (CENP-A) in humans and CID in drosophila] epigenetically determines and maintains centromeres (Malik and Henikoff, 2001; Dawe and Henikoff, 2006; Ekwall, 2007; Allshire and Karpen, 2008; Fachinetti et al., 2013). CENH3 contains a highly variable $\mathrm{N}$-terminal tail and a relatively conserved histone fold domain (HFD) (Ravi et al., 2010; Lermontova et al., 2014). The majority of diploid plant species have been shown to encode a single CENH3 gene (Zhong et al., 2002). However, more than one copy (alpha and beta) of the gene per genome are present in some species like wheat, barley, Arabidopsis halleri and A. lyrata (Kawabe et al., 2006; Sanei et al., 2011; Yuan et al., 2015).

The majority of cultivated bananas exist as allo- or autopolyploids and a variety of $C E N H 3$ isoforms are presumed to coexist in the nucleus. Polyploidization brings together multiple gene copies within the same background and can result in additive or non-additive gene expression leading to biased or unbiased homeolog expression (Pignatta and Comai, 2009; Hui et al., 2010; Rapp et al., 2010; Yoo et al., 2013). Unlike many diploid species where a single copy of CENH3 gene is encoded, multiple copies have been observed in newly synthesized allopolyploids of rice, wheat, brassica, and pea (Hirsch et al., 2009; Hui et al., 2010; Wang et al., 2011; Neumann et al., 2012; Yuan et al., 2015). CENH3 variants have also been characterized in wild and cultivated carrots (Dunemann et al., 2014) and in stable polyploids of different angiosperms (Masonbrink et al., 2014). Multiple CENH3 copies observed in polyploids might result from coming together of single-CENH3expressing genomes or multiple-CENH3 expressing progenitor genomes. Crosses of diploid parents encoding multiple CENH3 transcripts have resulted in stable hybrids. For example, in stable hybrids from Hordeum vulgare $\times H$. bulbosum crosses, both alpha and beta CENH3 variants from $H$. vulgare were incorporated into the centromeric nucleosomes of the hybrid. In contrast, a hybrid of $H$. bulbosum $\times$ Triticum aestivum incorporated the $H$. bulbosum $C E N H 3$ variant $\mathrm{Hb} \alpha \mathrm{CENH} 3$ only (Sanei et al., 2011).

Unlike stable hybrids, embryos derived from unstable crosses have been observed to undergo uniparental genome elimination, resulting in haploids carrying genetic material from only one parent (Ravi and Chan, 2010; Sanei et al., 2011; Seymour et al., 2012; Maheshwari et al., 2015). The genome of H. bulbosum in embryos from $H$. vulgare $\times H$. bulbosum crosses for example was completely lost within 5-9 days post-fertilization. Despite elimination of the $H$. bulbosum genome later in post-zygotic mitosis, $H$. vulgare $\times H$. bulbosum unstable crosses have been observed to transcribe CENH3 transcript variants from both parents (Sanei et al., 2011). In A. thaliana, uniparental genome elimination was also observed in offspring from crosses between mutant 'haploid inducer' (parent with modified CENH3) and wild-type (carrying wild-type CENH3 version) (Ravi and Chan,
2010). The modification of CENH3 in this case was generated by replacing the $\mathrm{N}$-terminal tail with that of the variant H3.3 and tagging it with GFP. Apart from obtaining haploids in these crosses, novel genetic rearrangements were observed (Maheshwari et al., 2015). Currently, there are efforts undergoing to transfer this technology to many crops including banana (Comai, 2014). Crosses of $A$. thaliana null-mutants carrying gene constructs expressing $C E N H 3$ from distant species to plants wildtype for $\mathrm{CENH} 3$ have also resulted in haploids (Maheshwari et al., 2015). Furthermore, uniparental genome elimination has been observed in crosses of wild-type $A$. thaliana plants to null mutants complemented with $\mathrm{CENH} 3$ carrying missense point mutations in conserved regions of the HFD (Kuppu et al., 2015).

Banana breeding involves crossing of tetraploids to diploids to give triploids and this may add into the complexity of the space CENH3 exists. Therefore, it would be interesting and useful to understand $\mathrm{CENH3}$ dynamics in cultivated polyploids and their diploid progenitors. Furthermore, a clear understanding of CENH3 behavior in cultivated crops like banana is essential if breeding tools such as $C E N H 3$-based haploid technology are to be effectively applied (Britt and Kuppu, 2016). Therefore, in this study the expression of $\mathrm{CENH} 3$ was characterized in cultivated triploid and wild-type diploid progenitor bananas. The existence and evolutionary relationships of CENH3 SNPs and/or splice variants as well as their predicted secondary folding of protein were analyzed.

\section{MATERIALS AND METHODS}

\section{Plant Materials}

Six banana genotypes including wild diploids 'Calcutta 4' (AA) and 'Zebrina GF' (AA) both from the species Musa acuminata, the species $M$. balbisiana $(\mathrm{BB})$ and cultivated triploids 'Sukali Ndiizi' (AAB), 'Pisang Awak' (ABB) and 'Gros Michel' (AAA) were used in this study. All plant materials used were obtained from in vitro collection at IITA Kenya.

\section{Identification of Genomic Sequence of Banana CENH3}

To identify putative genomic sequence of banana CENH3, a nucleotide BLAST (BLASTN) analysis was performed using genomic sequence of $A$. thaliana CENH3 (At1g01030) against the whole-genome shotgun contigs (wgs) of $M$. acuminata (tax id: 4641) for "somewhat similar sequences". In order to identify the exact genomic region of $C E N H 3$, consensus sequences from conserved regions at the beginning and end of selected monocot CENH3 CDSs were mapped to the BLASTN hit results. The conserved consensus, which we considered as representative $\mathrm{CENH} 3$ 'landmark' regions for monocots, were obtained by aligning sequences of CENH3 from the monocots Zea mays (NM_001112050), H. vulgare (JF419328), T. aestivum (JF969285.1) and Oryza sativa (AY438639.1). To identify the genomic regions of the CENH3 from $M$. acuminata, BLASTN hits, we mapped the CENH3 'landmarks' and regions with $>75 \%$ nucleotide identities were selected. The primers CENH3_END_F (GGCGAGAACGAAGCATC) and 
CENH3_END_R (TCACCAATGTCTTCTTCCTCC) were designed to amplify the CDS (from the beginning to the end of the coding region) derived from in silico analysis of the putative banana genomic sequence (Accession: CAIC01023700).

\section{RNA Extraction and RT-PCR}

Total RNA was extracted from $100 \mathrm{mg}$ of young incompletely open leaves. Extraction was performed using RNeasy ${ }^{\circledR}$ plant mini kit (Hilden, Germany) as per the manufacturer's protocol except for the elution volume which was reduced to $40 \mu \mathrm{l}$. Genomic DNA contamination was removed from the extracted RNA through DNase I (Thermo Scientific, Waltham, MA, United States) treatment by incubating at $37^{\circ} \mathrm{C}$ for $30 \mathrm{~min}$ and then terminating the reaction by adding $1 \mathrm{mM}$ EDTA and heating at $70^{\circ} \mathrm{C}$ for $5 \mathrm{~min}$. RNA quality and quantity were checked using a NanoDrop ${ }^{\mathrm{TM}} 2000$ (Thermo Scientific, Waltham, MA, United States) spectrophotometer.

First strand cDNA was synthesized from $1 \mu \mathrm{g}$ of DNA-free total RNA with random hexamer primers using maxima first strand reverse transcriptase kit (Thermo Scientific, Waltham, MA, United States). Two independent cDNA synthesis reactions were performed for each of the genotype.

The CENH3 transcripts were amplified from cDNA in a total of six PCR reactions (three reactions for each of the two cDNA synthesis) per genotype. Each PCR reaction was performed in a $20 \mu \mathrm{l}$ volume, which contained $50 \mathrm{ng}$ of cDNA template, $1 \mathrm{x}$ Q5 reaction buffer containing $2.5 \mathrm{mM} \mathrm{MgCl}_{2}, 500 \mu \mathrm{M}$ of each dNTP, $10 \mu \mathrm{M}$ each of CENH3 primers (CENH3_END_F and CENH3_END_R) and 1unit of Q5 high fidelity DNA polymerase (New England Biolabs, MA). The reactions were performed in an ABI 9700 PCR machine with the conditions set at initial denaturation of $98^{\circ} \mathrm{C}$ for $4 \mathrm{~min}, 35$ cycles of $98^{\circ} \mathrm{C}$ for $15 \mathrm{~s}, 66^{\circ} \mathrm{C}$ for $30 \mathrm{~s}$ and $72^{\circ} \mathrm{C}$ for $45 \mathrm{~s}$ and a final extension at $72^{\circ} \mathrm{C}$ for $10 \mathrm{~min}$. An aliquot of PCR product $(2 \mu \mathrm{l})$ was run on a $1.5 \%$ agarose gel stained with GelRed (Biotium, CA) to confirm amplification. For PCR reactions in each genotype that had observable band(s) on agarose gel, the remainder $(18 \mu \mathrm{l}) \mathrm{PCR}$ product was purified using Bioneer PCR purification kit (Daeongeon, South Korea) and eluted in $15 \mu \mathrm{l}$ water.

\section{Cloning and Sequencing of CENH3 Genes}

Purified PCR products were cloned into pJET 1.2 cloning vector (Thermo Scientific, MA) and transformed into competent Escherichia coli $(\mathrm{DH} 5 \alpha)$ cells using heat shock method. The transformed $E$. coli colonies were selected on Luria Bertani (LB) agar (10 g/l Tryptone, $5 \mathrm{~g} / \mathrm{l}$ Yeast extract, $10 \mathrm{~g} / \mathrm{l} \mathrm{NaCl}$, $15 \mathrm{~g} / \mathrm{l}$ Agar, $\mathrm{pH} 7.5$ ) containing $50 \mathrm{mg} / \mathrm{L}$ ampicillin. One to 10 transformed colonies from each PCR reaction were screened for presence of the insert by colony-PCR. A maximum of 60 colonies were screened for each genotype. The primer pairs $\mathrm{pJET}$ 1.2_F: CGACTCACTATAGGGAGAGCGGC and pJET 1.2_R: AAGAACATCGATTTTCCATGGCAG were used for colony PCR. Colonies with amplicon sizes $>200$ bp were cultured in LB broth medium overnight at $37^{\circ} \mathrm{C}$ and plasmid DNA extracted using Qiagen plasmid miniprep kit. Each clone with product
$>200$ bp was sequenced bi-directionally in three replicates using the primers pJET 1.2_F and Pjet 1.2_R. Sequencing was performed on ABI 3130 analyzer (Applied Biosystems, Foster City, CA, United States) using BigDye Terminator Kit version 3.1.

\section{Sequence Analysis and Multiple Alignments}

Sequences were analyzed in Geneious version 7.1 (Biomatter, NZ) (Kearse et al., 2012) by manually checking the quality of the chromatograms. Sequences with quality above 50\% (based on Phred values) across the entire sequence length were used for analysis. Sequences were further screened and 'dirty' sections at the ends were manually trimmed to retain only high quality regions. Sequences within any of the six genotypes that were independently derived (those obtained from amplification of independently synthesized cDNA transcripts) and had 100\% similarity were considered to represent the same transcript.

Multiple alignments of amino acids were conducted among translated banana CENH3 sequences and monocots (Z. mays, T. aestivum, O. sativa, and $H$. vulgare) and dicots (A. thaliana and Brassica rapa) in MUSCLE as implemented in Geneious version 7.1 using default parameters. Phylogenetic trees comparing transcript sequences were drawn in the software "Molecular and Evolutionary Genetic Analysis" (MEGA) version 6.0 (Tamura et al., 2013) based on only the conserved tail sections and entire HFD region.

\section{Identification of Exon/Intron Structures}

Since the banana CENH3 from the genotypes used in this study had not been sequenced previously, splicing patterns for the transcript sequences were predicted by aligning them to the then available banana genomic sequence (accession number: CAIC01023700 positions 70772 to 76310) from M. acuminata genotype 'DH Pahang' using the program Splign (Kapustin et al., 2008).

\section{Protein Structure Modeling}

Secondary structures of proteins were predicted using the original Garnier Osguthorpe Robson algorithm (GOR I) provided by the European Molecular Biology Open Software Suite (EMBOSS) 6.5.7 (Rice et al., 2000) and implemented in Geneious version 7.1.9 as garnier tool (Kearse et al., 2012). Predicted protein structures from transcripts of different length, SNP and splice were visually compared to determine any variation in their secondary folding.

\section{RESULTS}

\section{Identification of Genomic Sequence of Banana CENH3}

To identify CENH3 genomic sequence from completely sequenced banana genome [doubled haploid (DH) genotype 'DH Pahang' ('Malaccensis' group)] (Hont et al., 2012), a BLASTN was performed for 'somewhat' similar targets using A. thaliana CENH3 to query $M$. acuminata whole-genome 
contigs. This search resulted in a total of 46 hits (Additional File S1). To identify the exact banana CENH3 genomic region(s), conserved consensus sequences at the beginning (ATG GCSMGMACSAAGCAYCCGGCSGTGMGSAARAGC) and end (GCAAGGCGWATMGGAGGRAGRAGRCATTGGTGATGA) of CENH3 CDSs from four monocotyledonous plants (rice, maize, barley, and millet), referred as monocot CENH3 'landmarks', were searched within the 46 BLASTN hits. A search for these consensus sequences within the 46 BLASTN hit revealed an $82 \mathrm{~Kb}$ contiguous sequence (GenBank accession number: CAIC01023700) as containing the putative banana CENH3 genomic region. The exact location of the sequence within the $82 \mathrm{~Kb}$ contig CAIC01023700 was from positions 70772 to 76310 resulting in a 5538 bp long sequence.

\section{Banana CENH3 Sequences and Expressed Variants}

In an effort to identify banana CENH3 transcripts in each of the six banana genotypes, PCR products from amplification of cDNA template obtained from two independent synthesis reactions were cloned and sequenced. One to seven unique transcripts were obtained per genotype by sequencing of the multiple clones. The multiple clones sequenced were derived from three independent PCR amplifications of the two cDNA templates for a maximum of six reactions per genotype (Table 1). The genotype 'Calcutta 4' and 'M. balbisiana' had only one unique sequence each, where as 'Gros Michel' and 'Pisang Awak' had two unique sequences, 'Zebrina GF' had four and 'Sukali Ndiizi' had seven unique sequences (Table 1). All unique cDNA sequences from this study were deposited in GenBank (Table 1). The transcripts obtained were of variable lengths $(471,477,504,591$, and 760 bp). The open reading frames of the cDNA sequences encoded proteins of about 156-167 amino acids. The CENH3 sequence from 'Calcutta 4' (KT600803) was used as a reference as it had 100\% identity to the exons of the publicly available genomic sequence of banana genotype 'DH Pahang' (GenBank accession number CAIC01023700 position 70772 to 76310 ). The protein translation of the 'Calcutta 4' cDNA sequence resulted in a 167 amino acid long protein.

Based on similarity of conserved cDNA regions (partially in the tail and entire HFD region), the CENH3 sequences were clustered into three major groups denoted as MusaCENH3-1A (transcripts of $M$. balbisiana-10, Gros Michel-2, Pisang Awak-4) MusaCENH3-1B (Gros Michel-1, Zebrina GF-6, 7, and 8, Calcutta 4-11, Pisang Awak-5, and Sukali Ndiizi-A, F, G and H) and MusaCENH3-2 (Sukali Ndiizi-B, C and E and Zebrina GF-9) (Figure 1 and Table 1). The transcripts within each group had slight variations mainly less than two SNPs. The first two groups (MusaCENH3-1A and MusaCENH3-1B) differed from MusaCENH3-2 with a $\mathrm{C}$ to $\mathrm{G}$ substitution within the HFD $\alpha-2$ helix region that resulted in alanine $(\mathrm{A})$ to proline $(\mathrm{P})$ substitution in the later. In addition to this HFD SNP, transcripts in MusaCENH3-2 group consistently had a 46 bp longer exon 1 than MusaCENH3-1A and MusaCENH3-1B and also lacked extra two exons (exons 2 and 3), which were otherwise present in MusaCENH3-1A and MusaCENH3-1B groups. The 46 bp extra length in exon 1 as well as lack of exons 2 and 3 in MusaCENH3-2 suggests that this is a different type of CENH3 in bananas. However, since we did not sequence the whole genome of the genotypes used in this study, we cannot definitively prove that the missing exons or $46 \mathrm{bp}$ extension are indeed different genes or splice variants (as suggested by alignment to the published sequence), but this seems likely and we will refer to them as such. There were also multiple SNPs within transcripts of each CENH3

TABLE 1 | Description of CENH3 transcripts from different genotypes of banana.

\begin{tabular}{|c|c|c|c|c|c|c|c|c|}
\hline Genotype & $\begin{array}{l}\text { Genomic } \\
\text { group }\end{array}$ & $\begin{array}{c}\text { Banana } \mathrm{CENH3} \\
\text { group }\end{array}$ & $\begin{array}{l}\text { Unique sequence } \\
\text { identifier }\end{array}$ & $\begin{array}{l}\text { Total number of } \\
\text { clones }\end{array}$ & $\begin{array}{c}\text { CDS } \\
\text { length }\end{array}$ & $\begin{array}{l}\text { Exon/Intron } \\
\text { Structure }\end{array}$ & $\begin{array}{c}\text { Functional } \\
\text { status }\end{array}$ & $\begin{array}{c}\text { Genbank } \\
\text { Accession Number }\end{array}$ \\
\hline Gros Michel & AAA & MusaCENH3-1B & 1 & 6 & 591 & $6 / 5$ & $\begin{array}{c}\text { Non- } \\
\text { functional }\end{array}$ & KP878227 \\
\hline Gros Michel & AAA & MusaCENH3-1A & 2 & 7 & 504 & $7 / 6$ & Functional & KP878231 \\
\hline Pisang Awak & $\mathrm{ABB}$ & MusaCENH3-1A & 4 & 5 & 504 & $7 / 6$ & Functional & KP878229 \\
\hline Pisang Awak & $\mathrm{ABB}$ & MusaCENH3-1B & 5 & 4 & 760 & $5 / 4$ & $\begin{array}{c}\text { Non- } \\
\text { functional }\end{array}$ & KP878228 \\
\hline Sukali Ndiizi & $\mathrm{AAB}$ & MusaCENH3-1B & $\mathrm{G}$ & 6 & 471 & $6 / 5$ & Functional & KP878221 \\
\hline Sukali Ndiizi & $\mathrm{AAB}$ & MusaCENH3-1B & A & 4 & 504 & $7 / 6$ & Functional & KP878225 \\
\hline Sukali Ndiizi & $\mathrm{AAB}$ & MusaCENH3-1B & $\mathrm{F}$ & 7 & 477 & $7 / 6$ & Functional & KP878222 \\
\hline Sukali Ndiizi & $\mathrm{AAB}$ & MusaCENH3-1B & $\mathrm{H}$ & 3 & 504 & $7 / 6$ & Functional & KP878226 \\
\hline Sukali Ndiizi & $\mathrm{AAB}$ & MusaCENH3-2 & B & 5 & 471 & $5 / 4$ & Functional & KP878238 \\
\hline Sukali Ndiizi & $\mathrm{AAB}$ & MusaCENH3-2 & $\mathrm{C}$ & 4 & 471 & $5 / 4$ & Functional & KP878236 \\
\hline Sukali Ndiizi & $\mathrm{AAB}$ & MusaCENH3-2 & $E$ & 4 & 471 & $5 / 4$ & Functional & KP878239 \\
\hline Zebrina GF & $\mathrm{AA}$ & MusaCENH3-1B & 6 & 7 & 504 & $7 / 6$ & Functional & KP878223 \\
\hline Zebrina GF & $\mathrm{AA}$ & MusaCENH3-1B & 7 & 9 & 504 & $7 / 6$ & Functional & KP878224 \\
\hline Zebrina GF & AA & MusaCENH3-1B & 8 & 5 & 504 & $7 / 6$ & Functional & KP878220 \\
\hline Zebrina GF & $\mathrm{AA}$ & MusaCENH3-2 & 9 & 3 & 471 & $5 / 4$ & Functional & KP878237 \\
\hline Musa balbisiana & $\mathrm{BB}$ & MusaCENH3-1A & 10 & 13 & 504 & $7 / 6$ & Functional & KT600804 \\
\hline Calcutta 4 & $A A$ & MusaCENH3-1B & 11 & 13 & 504 & $7 / 6$ & Functional & KT600803 \\
\hline
\end{tabular}




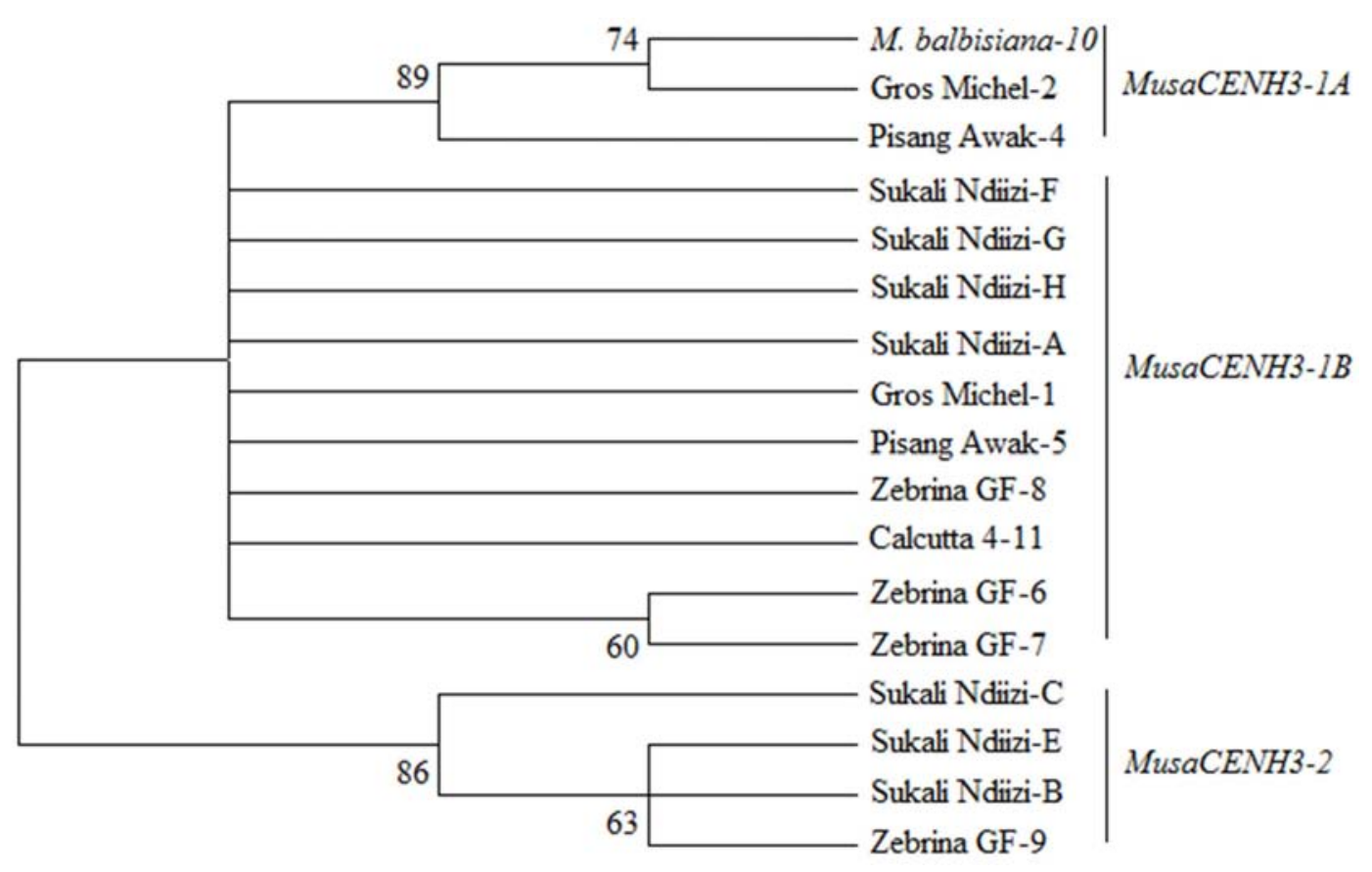

FIGURE 1| Phylogenetic tree of banana CENH3s. Unrooted Phylogenetic tree based on histone fold domain (HFD) and conserved CENH3 tail sections of six banana genotypes showing the MusaCENH3-1A, MusaCENH3-1B, and MusaCENH3-2 groups. Values at the root are bootstrap support values at 1000 replicates. The tree was drawn in MEGA 6 (Tamura et al., 2013).

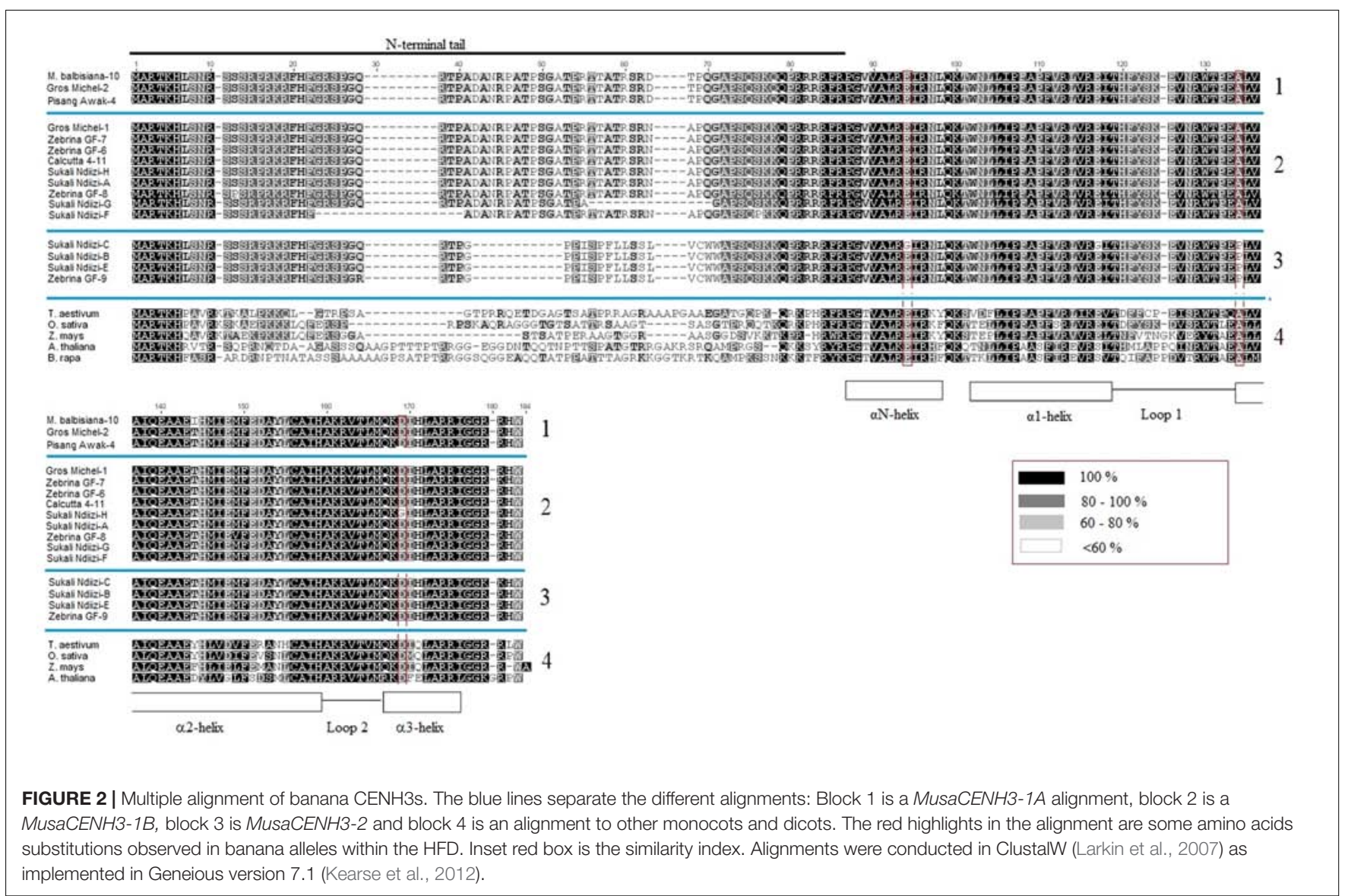




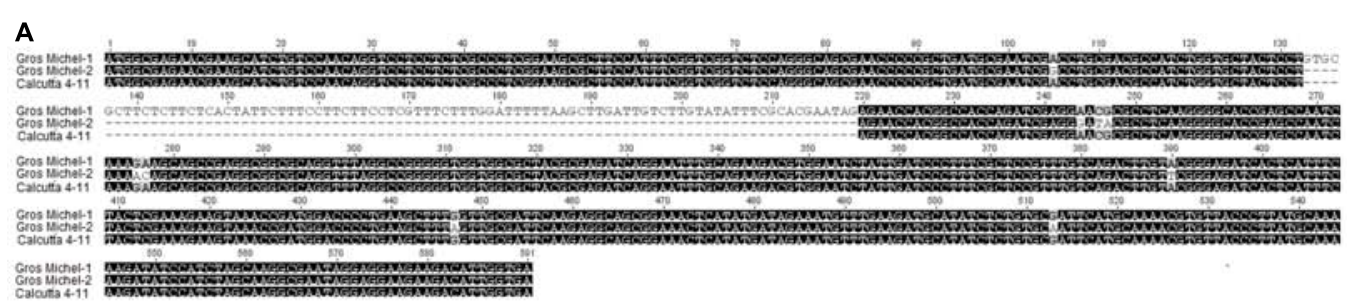

B

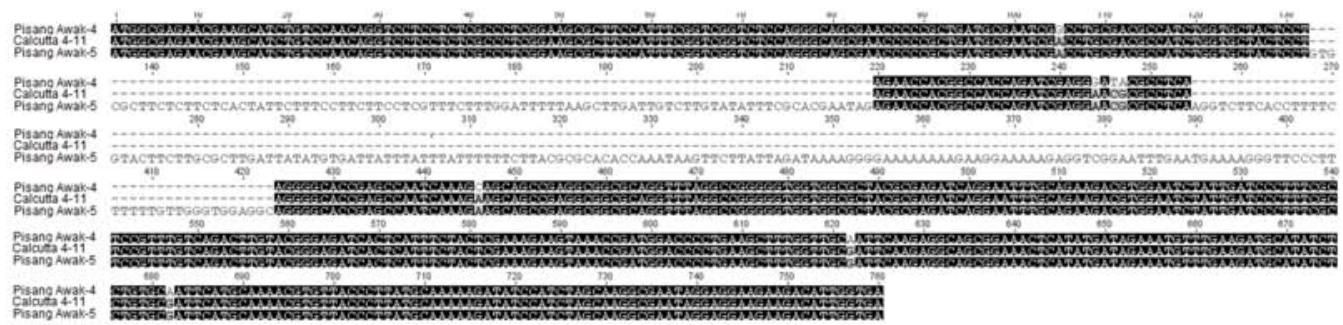

C

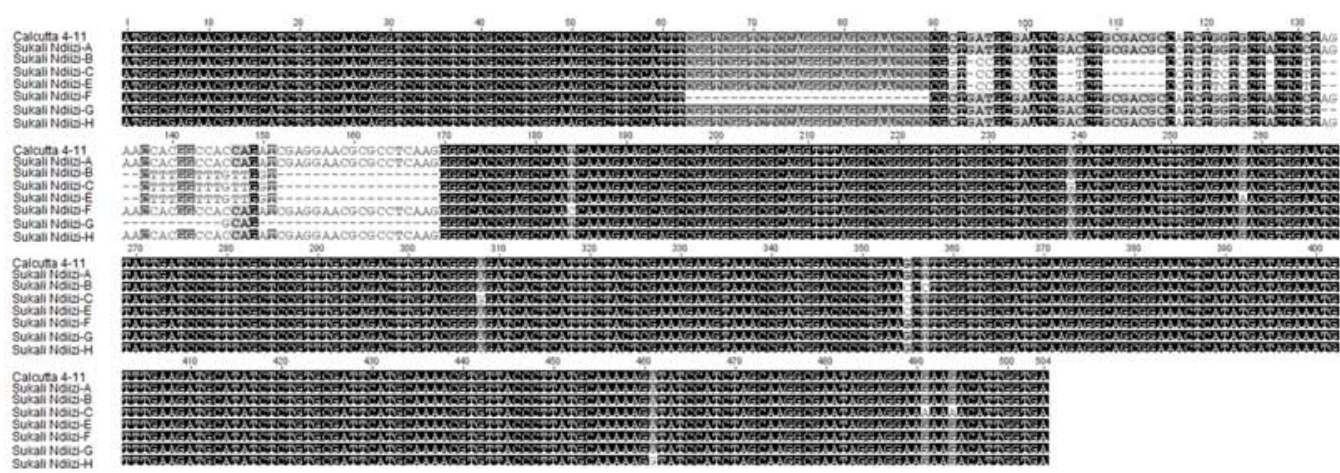

D

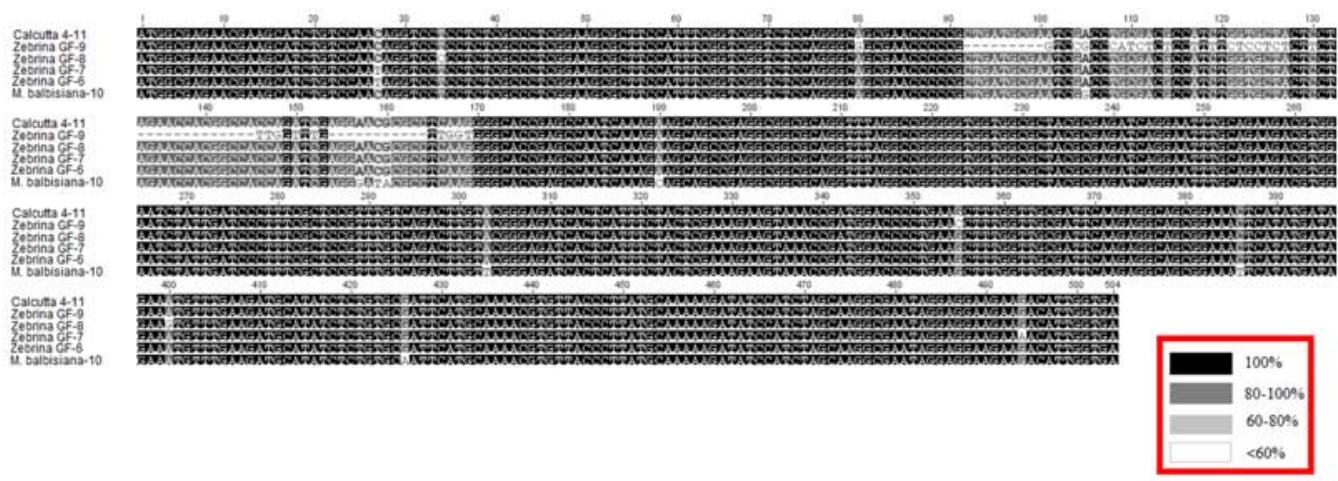

FIGURE 3 | Alignment of transcript variants to the reference transcript from diploid banana genotype 'Calcutta 4'. Blocks (A-D) are alignments of genotypes 'Gros Michel', 'Pisang Awak', 'Sukali Ndiizi', and a combination of 'Zebrina GF' and species 'Musa balbisiana' to 'Calcutta 4', respectively. Inset in red is the nucleotide alignment similarity index.

group, majority of which were within the HFD (Figures 2, 3). In comparison to CENH3s from other monocots and dicot species, banana sequences were observed to be highly variable within the tail region and conserved only in the loop 2 of the HDF (Figure 2).
The MusaCENH3-1A and MusaCENH3-1B groups were more similar to each other in both sequence and splicing in comparison to transcripts in group MusaCENH3-2. The MusaCENH3-1A and MusaCENH3-1B transcripts differed at five SNP sites (Figure 3), which resulted in one non-synonymous amino acid 
substitution (Figure 2). The MusaCENH3-1A was observed in both $\mathrm{A}$ and $\mathrm{B}$ genomes. The genotypes 'Zebrina GF' and 'Sukali Ndiizi' had the highest number of SNP variants observed (Table 2).

These three banana CENH3 groups differed in the number of exons as identified by alignment to the genomic sequence obtained through BLASTN analysis (CAIC01023700 position 70772-76310). The alignment confirmed that MusaCENH3-1A and $M u s a C E N H 3-1 B$ have seven exons whereas MusaCENH3-2 had five exons with exemptions of specific cases that differed due to exon skipping or intron retention. The MusaCENH3-1A and MusaCENH3-1B transcripts were 471 bp to 760 bp long while those in the MusaCENH3-2 group were 471 bp long. The three CENH3 groups had few SNPs among them that were observed mainly in transcripts from different genotypes.

To check the homology of banana CENH3 proteins to those of other plant species, banana translated protein sequences were aligned to monocot (T. aestivum, O. sativa, and Z. mays) and dicot species (A. thaliana and $B$. rapa). This alignment resulted in conserved $\alpha \mathrm{N}$-helix, $\alpha 1$-helix, $\alpha 2$-helix and $\alpha 3$-helix of the C-terminal, a specific loop 1 and a highly variable $\mathrm{N}$-terminal tail (Figure 2). The loop 1 and $\alpha 2$-helix of the C-terminal constitute the CENP-A targeting domain (CATD) and these two domains were found to be conserved in banana sequences except for two amino acid substitutions within the $\alpha 2$-helix in the sequences M. balbisiana-10 (alignment position 144) and Sukali NdiiziB, C, and E and in the Zebrina GF-9 (alignment position 134) (Figure 2).

\section{Variants in Autotriploid Genotype 'Gros Michel'}

'Gros Michel' had two variable and unique sequences as grouped in MusaCENH3-1A (Gros Michel-2) and MusaCENH3$1 B$ (Gros Michel-1). Over and above having the five SNPs that differentiated MusaCENH3-1A from MusaCENH3-1B, the transcript Gros Michel-1 had an 87 bp indel that resulted from retention of intron 2 and spanned alignment positions 133 to 219 (Figure 3A). This retained intron resulted in a frame shift and introduced a premature stop codon in the tail region (nucleotide position 219) rendering it non-functional. The transcript Gros Michel-2 differed to Calcutta 4 at nine SNPs and out of these, six were in the tail region. Two (alignment positions 105 and 276) out of six SNPs in the tail were synonymous substitutions. The other four SNPs were aligned at positions
244, 246, 247, and 277 resulted in a total of three amino acid substitutions.

\section{Variants in Allotriploid Genotype 'Pisang Awak'}

The allotriploid cultivated genotype 'Pisang Awak' had two unique transcripts that fell into the CENH3 groups MusaCENH3$1 A$ (Pisang Awak-5) and MusaCENH3-1B (Pisang Awak-4). Despite being in the MusaCENH3-1B group, the transcript Pisang Awak-5 had retained two introns (introns 2 and 3) (Figure 3B). These retained introns resulted in a non-functional protein by introducing multiple premature stop codons the first one at nucleotide position 219 in the tail region. The transcript Pisang Awak-4 carried two additional SNPs [one in the tail and one in HFD (alignment positions 105 and 622)] in addition to the five that allowed it to be grouped into the MusaCENH3-1B. Both SNPs were silent and did not result in any amino acid substitution.

\section{Variants in Allotriploid Genotype 'Sukali Ndiizi'}

The allotriploid genotype 'Sukali Ndiizi' had seven variants, four within MusaCENH3-1B group (transcripts Sukali Ndiizi-A, F, $\mathrm{G}$, and $\mathrm{H}$ ) and three within MusaCENH3-2 (Sukali Ndiizi-B, C, and E). Despite being in the same MusaCENH3-1B group, the sequences of Sukali Ndiizi-A and $\mathrm{H}$ differed to the Calcutta 4 at one SNP position each; positions 117 (A to C) and 461 (A to G) in Sukali Ndiizi-A and $\mathrm{H}$, respectively, with the latter resulting in a aspartic acid (D) to glycine (G) substitution in the protein sequence (Figures 2, 3C). The transcripts Sukali Ndiizi-F and G varied from each other with indels; Sukali Ndiizi-F had 27 bp indel (alignment position 63 - 89) as well as substitution from $\mathrm{T}$ to $\mathrm{C}$ at position 184 which resulted in a serine $(\mathrm{S})$ to proline $(\mathrm{P})$ substitution in the protein translation. The $27 \mathrm{bp}$ indel resulted in a shortened protein sequence with 158 amino acids due to a deletion in exon 1. The transcript Sukali Ndiizi-G on the other hand had a $37 \mathrm{bp}$ indel from alignment positions 133-169 (from skipping of exon 3 ), which resulted in alternative $3^{\prime}$ and $5^{\prime}$ splice sites. The two splice variations did not cause any shift in the reading frames and therefore resulted in functional proteins.

The transcripts falling within the MusaCENH3-2 group (Sukali Ndiizi-C, B, and E) were observed to be 471 bp long, which is 33 bp shorter than those in MusaCENH3-1A and MusaCENH3-1B and especially with Calcutta 4 (Figure 3C). The resultant proteins were all 156 amino acids long and

TABLE 2 | Minimum banana CENH3 allele and splice variants.

\begin{tabular}{|c|c|c|c|c|}
\hline Banana Genotype & $\begin{array}{l}\text { Minimum number of } \\
\text { SNP-allele variants }\end{array}$ & $\begin{array}{l}\text { Minimum number of } \\
\text { splice variants }\end{array}$ & Splicing mechanism (s) & $\begin{array}{l}\text { Splice variant CENH3 } \\
\text { group(s) }\end{array}$ \\
\hline Gros Michel & 2 & 1 & $7 / 6,6 / 5$ & MusaCENH3-1A and -1B \\
\hline Pisang Awak & 2 & 1 & $7 / 6,5 / 4$ & MusaCENH3-1A and -1B \\
\hline Sukali Ndiizi & 4 & 6 & $7 / 6,5 / 4$, and $6 / 5$ & MusaCENH3-1A, $-1 B$ and -2 \\
\hline Zebrina GF & 4 & 1 & $7 / 6$ and $5 / 4$ & MusaCENH3-1B and -2 \\
\hline Musa balbisiana & 1 & 0 & $7 / 6$ & MusaCENH3-1A \\
\hline Calcutta 4 & 1 & 0 & $7 / 6$ & MusaCENH3-1B \\
\hline
\end{tabular}


functional. Despite all the three transcripts being in the same group (MusaCENH3-2) they differed among themselves at six nucleotide positions, four of which resulted in amino acid substitutions at positions 94 and 117, 179 and 181 in Sukali Ndiizi-C (Figure 2).

\section{Variants in Diploid Banana 'Zebrina GF' and 'Musa balbisiana'}

The diploid banana genotype 'Zebrina GF' expressed transcripts that fell into both the MusaCENH3-1B (transcripts Zebrina GF6, 7, and 8) and MusaCENH3-2 (Zebrina GF-9) categories. The three transcripts in MusaCENH3-1B differed amongst themselves with four SNPs, three of which were non-synonymous substitutions at alignment positions 34 ( $\mathrm{T}$ to $\mathrm{C}$ ) and 400 (A to G) in Zebrina GF-8 and position 494 in Zebrina GF-7 (G to A) (Figure 3D). The only MusaCENH3-2 representative sequence in this genotype was transcript Zebrina GF-9, which was 471 bp encoding a 156 amino acid long protein. This transcript, like others in the same group from other cultivars, had an exon 1 that was 45 bp longer, the $\mathrm{C}$ to $\mathrm{G}$ substitution in the $\alpha-2$ helix and in addition an $A$ to $G$ non-synonymous substitution at alignment position 80 (Figure 3D) that resulted in glycine (Q) to argenine (R) substitution at protein alignment position 28 (Figure 2).

The diploid species ' $M$. balbisiana' had one unique 504 bp long sequence that encoded a 167 amino acid long protein. This transcript fell into the MusaCENH3-1A group and differed from others in the same group with one major non-synonymous
SNP site in the HFD that resulted in the substitution of the amino acid threonine (T) to isoleucine (I) at alignment position 144 .

\section{Exon/Intron Structures in Banana CENH3}

To get an insight into the splicing approaches and the intron/exon structures of the transcripts obtained and to also know if the differences in lengths of the transcripts were due to splicing variations, the unique banana CENH3 transcripts were mapped to genomic sequence of putative CENH3 from 'DH Pahang' (Figure 4 and Additional File S2). Three exon/intron structures $(7 / 6,6 / 5$, and 5/4) were observed, which were probably as a result of differences in splicing patterns (Figure 4). The 7 exon/6 intron structure was most frequently observed (10 transcripts out of 17 unique clones). This structure was observed in both diploid and triploid genotypes with three of the four transcripts from the diploid 'Zebrina GF' (Zebrina GF-6, 7, and 8), diploid 'Calcutta 4' and ' $M$. balbisiana', triploid genotype 'Pisang Awak' (Pisang Awak-4), in three of the seven sequences in the genotype 'Sukali Ndiizi' (Sukali Ndiizi-A, F, and H) and in one transcript from the autopolyploid 'Gros Michel' (Gros Michel-2) (Table 1).

The 5 exon/4 intron structure was observed in genotypes 'Pisang Awak' (Pisang Awak-5), 'Sukali Ndiizi' (Sukali Ndiizi-B, $\mathrm{C}$, and E) and the diploid 'Zebrina GF' (Zebrina GF-9) (Figure 4). This structure resulted from skipping of the second and the third exons in all sequences apart from Pisang Awak-5, which had this structure due to retention of introns two and three.

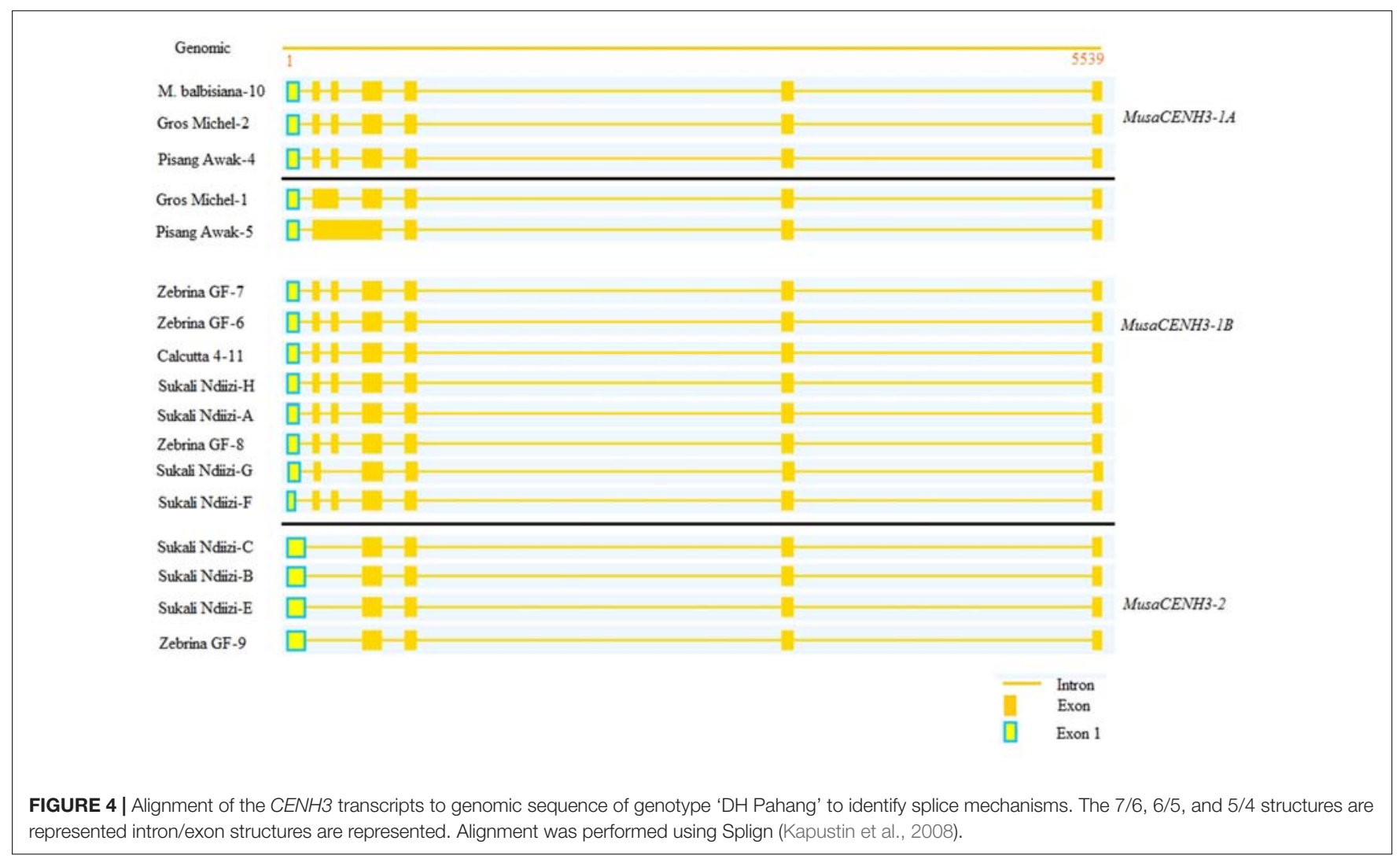


The 6 exon/5 intron pattern was observed in two transcripts of Gros Michel-1 and Sukali Ndiizi-G from the genotype 'Gros Michel' and 'Sukali Ndiizi', respectively (Figure 4). This structure was as a result of skipping of exon 2 for Sukali Ndiizi-G and retention of intron 2 in the transcript Gros Michel-1.

\section{Alternative Splicing of CENH3 in Banana}

Alternative splicing achieves diversity and novelty of proteins. Alternatively spliced variants were obtained based on deviations from splicing in their respective banana $C E N H 3$ groups. Four out of the seventeen unique transcripts were alternatively spliced with two of these resulting in unique proteins while the rest introduced premature stop codons. Some of the alternatively spliced transcripts also had SNP variations. Three alternative splicing approaches were observed: exon skipping, intron retention and alternate $3^{\prime}$ and $5^{\prime}$ splice site (Figure 4 and Additional File S2).

\section{Alternative Splicing by Exon Skipping}

Exon skipping was observed in only one transcript (Sukali Ndiizi-G) of the triploid cultivar 'Sukali Ndiizi'. This alternate splicing mechanism resulted from skipping of exon 3 and resulted in a functional transcript (Figure 4 and Additional File S2). Exon skipping resulted in shorter transcript length, where the transcript affected (Sukali Ndiizi-G) had a reduced length of 471 bp instead of the 504 bp in transcripts from the same CENH3 group.

\section{Alternative Splicing by Intron Retention}

Intron retention as an alternative splicing mechanism was observed in two transcripts (Gros Michel-1 and Pisang Awak-5), which retained one and two introns, respectively (Figure 4). The intron retention resulted in non-functional proteins due to introduction of at least one stop codon in either of the two transcripts. The transcript Pisang Awak-5 had five stop codons introduced, four in the tail and one in the HFD, whereas Gros Michel-1 only had one stop codon in the tail region.

\section{Splice Variation by Alternative Splice Site Selection}

The alternate $3^{\prime}$ and $5^{\prime}$ splice site selection resulted in variation in the length of exon 1 (Figure 4). Partial deletion of a $27 \mathrm{bp}$ segment from positions 63-89 of exon 1 was observed in Sukali NdiiziF. This deletion resulted in a change of the splice junction from CCCC/GGTC to TTTC/GGTC resulting in a change in splice sites. The transcript Sukali Ndiizi-G was also observed to have a different splice site selection by retaining the nucleotide $\mathrm{G}$ from intron 2 (Exon 3 was skipped) and retaining the nucleotide $\mathrm{C}$ of intron 3.

\section{Secondary Structure Prediction}

There was general conservation in predicted secondary structure within each of the banana CENH3 groups, although slight variations at specific sections were observed (Figure 5). The MusaCENH3-1A and MusaCENH3-1B had similar predicted secondary folding and varied in the second and the third last turns of the $\mathrm{N}$-terminal tail where they were merged into one due to the lack of the predicted intervening beta sheet in the MusaCENH3-1A group. The secondary structures in the MusaCENH3-2 had more structural variation in comparison to the MusaCENH3-1A and MusaCENH3-1B. The structural modifications included addition, loss, elongation or shortening of coils, turns, $\alpha$-helices and $\beta$-sheets. Splice variations affecting the tail region resulted in loss of $\alpha$-helices and beta strands, coils and turns in Sukali Ndiizi-G and F (Figure 5). The CENH3 proteins for Sukali Ndiizi-C, B, E, and Zebrina GF-9 also gained and lost domains within the tail region. The major form of variation observed within the HFD was point mutations some of which resulted in non-synonymous substitution. These substitutions resulted in elongations and/or shortening of some predicted secondary structures. The Proline (P) to Alanine (A) substitution within the HFD in Sukali Ndiizi-C, B, E, and Zebrina GF-9 resulted in an elongated loop 1 and a shortened $\alpha 2$ helix a clear structural feature unique to the MusaCENH3-2 CENH3 groups

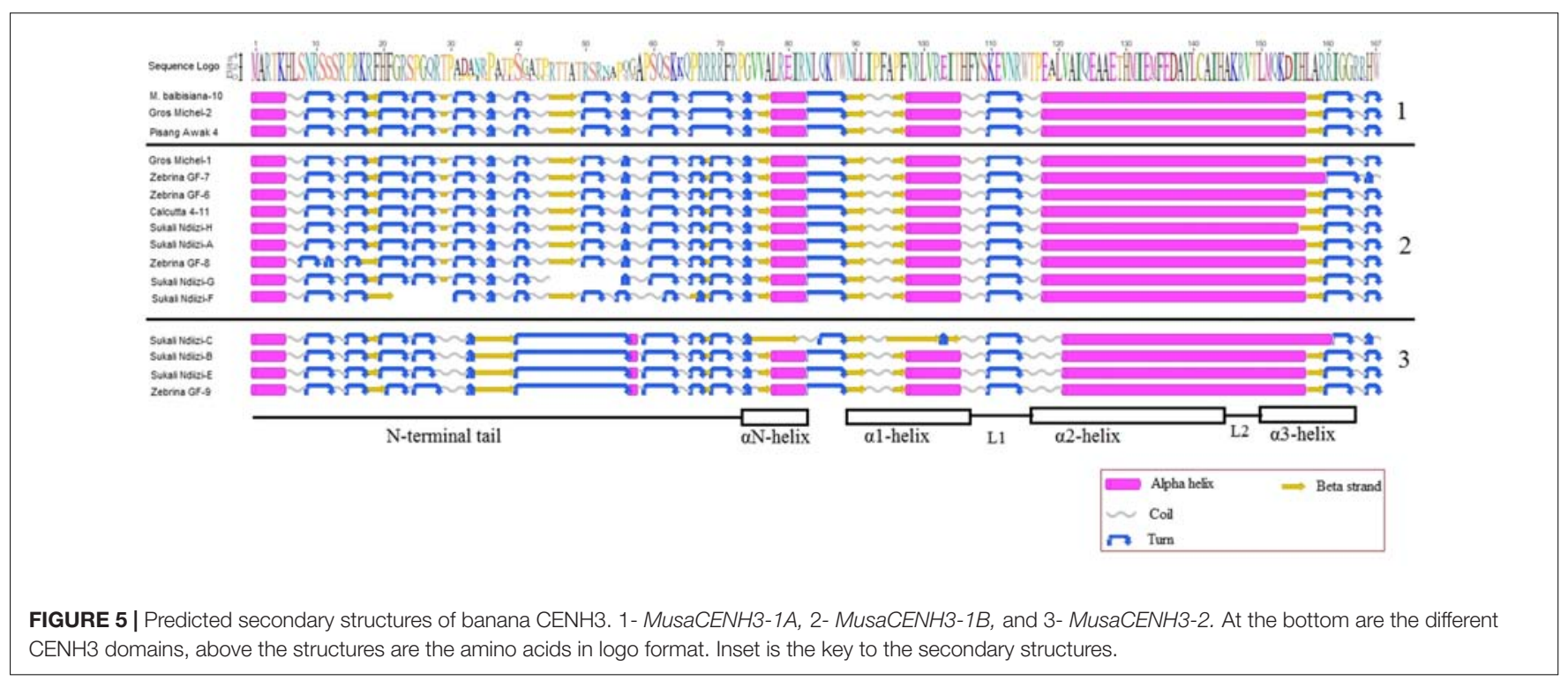


(Figure 5). The substitutions of glutamine (E) with glycine (G) at two different positions in Sukali Ndiizi-C resulted in structural changes within the $\alpha \mathrm{N}$ - and $\alpha 1$-helices (Figure 5).

\section{DISCUSSION}

In this study, we observed that $\mathrm{CENH} 3$ in diploid and triploid bananas exists as single or multiple allele variants depending on the genotype. The variants were SNPs in both the tail and the HFD region of CENH3. The non-synonymous SNPs resulted in modification of the predicted secondary structures of proteins. The majority of splice variants (apart from two) were predicted to translate in-frame. These splice variations only affected the tail region of CENH3. The presence of multiple CENH3 SNP-alleles in a diploid genotype like 'Zebrina GF' suggests that bananas maybe carrying more than one $\mathrm{CENH} 3$ gene per genome.

Cultivated bananas are mainly triploids. Banana breeding involves crossing fertile triploids with diploids to get tetraploids which are then crossed to diploid accessions to give triploid cultivars (Pillay et al., 2004). In other plant species, the presence of different CENH3s from different parents in embryos has been observed to result in uniparental genome elimination, aneuploids, or stable hybrids (Ravi and Chan, 2010; Kuppu et al., 2015; Maheshwari et al., 2015; Tan et al., 2015; Kelliher et al., 2016). The focus in banana breeding programs is to first establish tetraploids and then use them to develop triploids. It has been suggested that crosses between diploids and triploids result in viable diploids (De Langhe et al., 2010). Crosses between A. thaliana wild-type parents and pollen donors carrying specific point mutations within the HFD resulted in uniparental genome elimination- with loss of the genome derived from the mutant line (Kuppu et al., 2015). Two out of the five point mutations that resulted in uniparental genome elimination in Arabidopsis were within the centromere targeting domain (CATD). Some of the SNPs in our study were observed to be within the conserved HFD domains including $\alpha 2$ - and $\alpha 3$-helices. Furthermore, mutations of CENP-A (CENH3 of humans) residues resulted in reduced retention of CENP-A in centromere of human cells and this was due to the effect on $\alpha 2$-helix length which plays a key role of maintaining orientation at nucleosome entry and exit (Tachiwana et al., 2011). The SNPs were within the CATD and these resulted in a predicted shortening of the $\alpha 2$-helix. These CATD SNP variations can result in CENH3 nucleosome instability and may affect crosses of bananas having $\mathrm{CENH} 3 \mathrm{~s}$ with variation at these positions. It would be interesting to know if multiple CENH3s affect banana breeding and if they do, then it may be important to consider $\mathrm{CENH} 3$ type when choosing parents for crossing.

We observed three CENH3 variants in both diploid and triploid bananas, which differed at the tail region and have SNPs in the HFD region. The presence of these three variants in a diploid line indicates presence of more than one CENH3 in a single banana genome. Alpha and beta CENH3 variants in wheat were observed to have different functional roles. Reduced expression of alpha version resulted in extreme dwarfing and weakened root system whereas reduced expression of the beta version resulted in reduced plant height and reproductive fitness leading to the conclusion that the two versions are involved in plant development and reproductive development, respectively (Yuan et al., 2015). Although this study did not explore the functions of the banana CENH3 variants, it would be worth conducting such studies in future to verify if the variants differ in functionality.

The presence of multiple CENH3 allele variants in a wild diploid banana (four in diploid 'Zebrina GF') corroborate the hypothesis that domestication of cultivated hybrids passed through intermediate hybrids (De Langhe et al., 2010). 'Zebrina GF' is a wild diploid that has been shown to segregate during crosses, an indication that it has a high degree of heterozygozity (personal communication from Professor Rony Swennen, Banana breeder at IITA and collector of this genotype).

The observation that alternative splicing of CENH3 in bananas only affected the N-terminal tail is consistent with those made in the angiosperms Oryza spp., Brassica spp., and Gossypium spp. (Wang et al., 2011; Masonbrink et al., 2014). It was interesting to observe that some of the splice variations resulted in transcripts that were translatable into proteins as these could further add into the diversity of banana CENH3s. However, it is not clear if the in-frame splice variants translate into proteins in vivo and whether they are loaded into the centromere. The role of the outof-frame variants is also not clear and future studies targeting the CENH3 splice variants and their proteins (if translated) are required to identify their role(s) and fate.

The variations observed in the three main banana CENH3 groups were observed to affect the predicted secondary structures of the respective proteins. This is interesting considering that crosses of Arabidopsis null mutant lines complemented with a $\mathrm{CENH} 3$ version in which tail was replaced with that of histone H3.3 and GFP-tagged to wild-type resulted in uniparental genome elimination (Ravi and Chan, 2010). The highly variable $\mathrm{N}$-terminal tail of the CENH3 indicates its role in the evolving centromeric satellites (Hui et al., 2010; Ravi et al., 2010; Hayden and Willard, 2012) or affecting the targeting of centromeres that might be a mode of bringing in new $\mathrm{CENH} 3$ proteins in response to increased centromere size (Masonbrink et al., 2014).

The frequency of non-synonymous SNPs within each of the banana CENH3 groups was observed to be higher within the HFD region, while the frequency of both synonymous and non-synonymous SNPs between different CENH3 groups was higher in the tail region. A study on evolution of CENH3 in drosophila observed that the frequency of interspecific CENH3 polymorphisms were higher in the tail than the HFD although the ratios of such changes were lower within the same species (Malik and Henikoff, 2001). One of the CENH3 groups (MusaCENH3-1A) was observed to be specific to the diploid Musa species 'M. balbisiana' and differed from other CENH3 groups with non-synonymous substitutions, majority of which were in the tail region. Majority of the non-synonymous SNPs in transcripts within banana CENH3 group were observed to be within the CATD, which may affect CENH3 targeting to the centromere because the CATD specifically the loop 1 has been shown to be involved in localization (Dalal et al., 2007).

The number of CENH3 exons and introns in the respective exon/intron structures has been found to vary in different plant 
species. Seven CENH3 transcripts obtained from five rice species were observed to have 7 exons and 6 introns despite having different CDS lengths (Hirsch et al., 2009). In carrots, a similar structure of 7 exons and 6 introns was observed while in brassica two different structures were observed in CENH3s of varying lengths, one with 7 exons/ 6 introns and second with 9 exons/8 introns structure. In this study, three exons/introns structures $(7 / 6,6 / 5$, and $5 / 4)$ were observed in bananas. The $7 / 6$ and the $5 / 4$ exon/intron structures were found in both diploids and triploids, however, 7/6 was most predominant. The 6 exons/5 introns structure was only observed in triploid bananas and this mechanism resulted in functional and non-functional transcripts. In this analysis, it is clear that there was more bias toward having a 7 exons/6 introns structure whereas the 5 exons/4 introns structure was minor and the $6 / 5$ structure was a result of failure of the $7 / 6$ to splice correctly.

This study provided insight into how CENH3 is expressed in diploid and triploid bananas. Additional genotypes including tetraploids should be included in future studies. Due to the emergence of CENH3-based breeding techniques, the knowledge obtained here indicates that checking the CENH3 type may be used as a criterion in selection of parents for banana breeding.

\section{AVAILABILITY OF SUPPORTING DATA}

Gene sequences for banana CENH3 obtained in this study were deposited in the GenBank and the accession numbers are provided in this manuscript, all other supporting data is providing as additional files.

\section{REFERENCES}

Allshire, R. C., and Karpen, G. H. (2008). Epigenetic regulation of centromeric chromatin: old dogs, new tricks? Nat. Rev. Genet. 9, 923-937. doi: 10.1038/ $\operatorname{nrg} 2466$

Black, B. E., and Cleveland, D. W. (2011). Epigenetic centromere propagation and the nature of CENP-A nucleosomes. Cell 144, 471-479. doi: 10.1016/j.cell.2011. 02.002

Britt, A. B., and Kuppu, S. (2016). Cenh3: an emerging player in haploid induction technology. Front. Plant Sci. 7:357. doi: 10.3389/fpls.2016.00357

Comai, L. (2014). Genome elimination: translating basic research into a future tool for plant breeding. PLoS Biol. 12:e1001876. doi: 10.1371/journal.pbio.1001876

Dalal, Y., Furuyama, T., Vermaak, D., and Henikoff, S. (2007). Structure, dynamics, and evolution of centromeric nucleosomes. Proc. Natl. Acad. Sci. U.S.A. 104, 15974-15981. doi: 10.1073/pnas.0707648104

Dawe, R. K., and Henikoff, S. (2006). Centromeres put epigenetics in the driver's seat. Trends Biochem. Sci. 31, 662-669. doi: 10.1016/j.tibs.2006.10.004

De Langhe, E., Hřibová, E., Carpentier, S., Doleel, J., and Swennen, R. (2010). Did backcrossing contribute to the origin of hybrid edible bananas? Ann. Bot. 106, 849-857. doi: 10.1093/aob/mcq187

Dunemann, F., Schrader, O., Budahn, H., and Houben, A. (2014). Characterization of centromeric histone $\mathrm{H} 3$ (CENH3) variants in cultivated and wild carrots (Daucus sp.). PLoS ONE 9:e98504. doi: 10.1371/journal.pone.0098504

Ekwall, K. (2007). Epigenetic control of centromere behavior. Annu. Rev. Genet. 41, 63-81. doi: 10.1146/annurev.genet.41.110306.130127

Fachinetti, D., Folco, H. D., Nechemia-Arbely, Y., Valente, L. P., Nguyen, K., Wong, A. J., et al. (2013). A two-step mechanism for epigenetic specification of centromere identity and function. Nat. Cell Biol. 15, 1056-1066. doi: 10.1038/ ncb2805

\section{AUTHOR CONTRIBUTIONS}

$\mathrm{KM}$, LT, AB, and SC conceived the idea and designed the experiments. KM performed the experiments and wrote the manuscript. LT and $\mathrm{AB}$ supervised the experimentation. All authors contributed in interpreting the data. KM, AB, LT, NA, and $\mathrm{EN}$ contributed to reviewing and editing the manuscript.

\section{ACKNOWLEDGMENTS}

This work was supported by funding from National Science Foundation (NSF) and the Bill \& Melinda Gates Foundation (BMGF) under the program Basic Research Enabling Agricultural Development (BREAD) project number 1109882: Fast Breeding for Slow Cycling Crops: Doubled Haploids in Cassava and Banana/Plantain.

\section{SUPPLEMENTARY MATERIAL}

The Supplementary Material for this article can be found online at: http://journal.frontiersin.org/article/10.3389/fpls.2017.01034/ full\#supplementary-material

ADDITIONAL FILE S1 |BLAST hits on Musa acuminata whole genome shot gun contigs using $A$. thaliana CENH3 genomic sequence as the query.

ADDITIONAL FILE S2 | Alignment of banana CENH3 transcripts to the putative genomic sequence obtained using $A$. thaliana $C E N H 3$ genomic sequence to query $M$. acuminata whole genome contig.

Gent, J. I., Schneider, K. L., Topp, C. N., Rodriguez, C., Presting, G. G., and Dawe, R. K. (2011). Distinct influences of tandem repeats and retrotransposons on CENH3 nucleosome positioning. Epigenetics Chromatin 4, 3. doi: 10.1186/ 1756-8935-4-3

Hayden, K. E., and Willard, H. F. (2012). Composition and organization of active centromere sequences in complex genomes. BMC Genomics 13:324. doi: 10. 1186/1471-2164-13-324

Hirsch, C. D., Wu, Y., Yan, H., and Jiang, J. (2009). Lineage-specific adaptive evolution of the centromeric protein $\mathrm{CENH} 3$ in diploid and allotetraploid Oryza species. Mol. Biol. Evol. 26, 2877-2885. doi: 10.1093/molbev/msp208

Hont, D., Denoeud, F., Aury, J., Baurens, F., Carreel, F., Garsmeur, O., et al. (2012). The banana (Musa acuminata) genome and the evolution of monocotyledonous plants. Nature 488, 213-217. doi: 10.1038/nature11241

Hui, L., Lu, L., Heng, Y., Qin, R., Xing, Y., and Jin, W. (2010). Expression of CENH3 alleles in synthesized allopolyploid Oryza species. J. Genet. Genomics 37, 703-711. doi: 10.1016/S1673-8527(09)60088-6

Kapustin, Y., Souvorov, A., Tatusova, T., and Lipman, D. (2008). Splign: algorithms for computing spliced alignments with identification of paralogs. Biol. Direct 3:20. doi: 10.1186/1745-6150-3-20

Kawabe, A., Nasuda, S., and Charlesworth, D. (2006). Duplication of centromeric histone H3 (HTR12) gene in Arabidopsis halleri and A. lyrata, plant species with multiple centromeric satellite sequences. Genetics 174, 2021-2032. doi: 10.1534/genetics.106.063628

Kearse, M., Moir, R., Wilson, A., Stones-Havas, S., Cheung, M., Sturrock, S., et al. (2012). Geneious Basic: an integrated and extendable desktop software platform for the organization and analysis of sequence data. Bioinformatics 28, 1647-1649. doi: 10.1093/bioinformatics/bts199

Kelliher, T., Starr, D., Wang, W., McCuiston, J., Zhong, H., Nuccio, M. L., et al. (2016). Maternal haploids are preferentially induced by CENH3-tailswap 
transgenic complementation in maize. Front. Plant Sci. 7:414. doi: 10.3389/fpls. 2016.00414

Kuppu, S., Tan, E. H., Nguyen, H., Rodgers, A., Comai, L., Chan, S. W. L., et al. (2015). Point mutations in centromeric histone induce post-zygotic incompatibility and uniparental inheritance. PLoS Genet. 11:e1005494. doi: 10.1371/journal.pgen.1005494

Larkin, M. A., Blackshields, G., Brown, N. P., Chenna, R., Mcgettigan, P. A., McWilliam, H., et al. (2007). Clustal W and Clustal X version 2.0. Bioinformatics 23, 2947-2948. doi: 10.1093/bioinformatics/btm404

Lermontova, I., Sandmann, M., and Demidov, D. (2014). Centromeres and kinetochores of Brassicaceae. Chromosome Res. 22, 135-152. doi: 10.1007/ s10577-014-9422-z

Maheshwari, S., Tan, E. H., West, A., Franklin, F. C. H., Comai, L., and Chan, S. W. L. (2015). Naturally occurring differences in CENH3 affect chromosome segregation in zygotic mitosis of hybrids. PLOS Genet. 11:e1004970. doi: 10.1371/journal.pgen.1004970

Malik, H. S., and Henikoff, S. (2001). Adaptive evolution of Cid, a centromerespecific histone in Drosophila. Genetics 157, 1293-1298.

Malik, H. S., and Henikoff, S. (2009). Major evolutionary transitions in centromere complexity. Cell 138, 1067-1082. doi: 10.1016/j.cell.2009.08.036

Masonbrink, R. E., Gallagher, J. P., Jareczek, J. J., Renny-Byfield, S., Grover, C. E., Gong, L., et al. (2014). CenH3 evolution in diploids and polyploids of three angiosperm genera. BMC Plant Biol. 14:383. doi: 10.1186/s12870-014-0383-3

Neumann, P., Navrátilová, A., Schroeder-Reiter, E., Kobližková, A., Steinbauerová, V., Chocholová, E., et al. (2012). Stretching the rules: monocentric chromosomes with multiple centromere domains. PLoS Genet. 8:e1002777. doi: 10.1371/journal.pgen.1002777

Pignatta, D., and Comai, L. (2009). Parental squabbles and genome expression: lessons from the polyploids. J. Biol. 8, 43. doi: 10.1186/jbiol140

Pillay, M., Ssebuliba, R., Hartman, J., Vuylsteke, D., Talengera, D., and Tushemereirwe, W. (2004). Conventional breeding strategies to enhance the sustainability of Musa biodiversity conservation for endemic cultivars. Afr. Crop Sci. J. 12, 59-65. doi: 10.4314/acsj.v12i1.27663

Rapp, R. A., Haigler, C. H., Flagel, L., Hovav, R. H., Udall, J. A., and Wendel, J. F. (2010). Gene expression in developing fibres of Upland cotton (Gossypium hirsutum L.) was massively altered by domestication. BMC Biol. 8:139. doi: 10.1186/1741-7007-8-139

Ravi, M., and Chan, S. W. L. (2010). Haploid plants produced by centromeremediated genome elimination. Nature 464, 615-618. doi: 10.1038/nature 08842

Ravi, M., Kwong, P. N., Menorca, R. M. G., Valencia, J. T., Ramahi, J. S., Stewart, J. L., et al. (2010). The rapidly evolving centromere-specific histone has stringent functional requirements in Arabidopsis thaliana. Genetics 186, 461-471. doi: 10.1534/genetics.110.120337

Rice, P., Longden, I., and Bleasby, A. (2000). EMBOSS: the european molecular biology open software suite. Trends Genet. 16, 276-277. doi: 10.1016/j.cocis. 2008.07.002
Sanei, M., Pickering, R., Kumke, K., Nasuda, S., and Houben, A. (2011). Loss of centromeric histone $\mathrm{H} 3$ (CENH3) from centromeres precedes uniparental chromosome elimination in interspecific barley hybrids. Proc. Natl. Acad. Sci. U.S.A. 108, E498-E505. doi: 10.1073/pnas.1103190108

Seymour, D. K., Filiault, D. L., Henry, I. M., Monson-Miller, J., Ravi, M., Pang, A., et al. (2012). Rapid creation of Arabidopsis doubled haploid lines for quantitative trait locus mapping. Proc. Natl. Acad. Sci. U.S.A. 109, 4227-4232. doi: $10.1073 /$ pnas.1117277109

Tachiwana, H., Kagawa, W., Shiga, T., Osakabe, A., Miya, Y., Saito, K., et al. (2011). Crystal structure of the human centromeric nucleosome containing CENP-A. Nature 476, 232-235. doi: 10.1038/nature10258

Talbert, P. B., Bryson, T. D., and Henikoff, S. (2004). Adaptive evolution of centromere proteins in plants and animals. J. Biol. 3, 18. doi: 10.1186/jbiol11

Tamura, K., Stecher, G., Peterson, D., Filipski, A., and Kumar, S. (2013). MEGA6: Molecular Evolutionary Genetics Analysis version 6.0. Mol. Biol. Evol. 30, 2725-2729. doi: 10.1093/molbev/mst197

Tan, E. H., Henry, I. M., Ravi, M., Bradnam, K. R., Mandakova, T., Marimuthu, M. P. A., et al. (2015). Catastrophic chromosomal restructuring during genome elimination in plants. Elife 4:e06516. doi: 10.7554/eLife.06516

Verdaasdonk, J., and Bloom, K. (2011). Centromeres: unique chromatin structures that drive chromosome segregation. Nat. Rev. Mol. Cell Biol. 12, 320-332. doi: 10.1038/nrm3107

Wang, G., He, Q., Liu, F., Cheng, Z., Talbert, P., and Jin, W. (2011). Characterization of CENH3 proteins and centromere-associated DNA sequences in diploid and allotetraploid Brassica species. Chromosoma 120, 353-365. doi: 10.1007/s00412-011-0315-z

Yoo, M.-J., Szadkowski, E., and Wendel, J. F. (2013). Homoeolog expression bias and expression level dominance in allopolyploid cotton. Heredity (Edinb). 110, 171-180. doi: 10.1038/hdy.2012.94

Yuan, J., Guo, X., Hu, J., Lv, Z., and Han, F. (2015). Characterization of two CENH3 genes and their roles in wheat evolution. New Phytol. 206, 839-851. doi: $10.1111 /$ nph. 13235

Zhong, C., Marshall, J., and Topp, C. (2002). Centromeric retroelements and satellites interact with maize kinetochore protein CENH3. Plant Cell 14, 28252836. doi: 10.1105/tpc.006106

Conflict of Interest Statement: The authors declare that the research was conducted in the absence of any commercial or financial relationships that could be construed as a potential conflict of interest.

Copyright (c) 2017 Muiruri, Britt, Amugune, Nguu, Chan and Tripathi. This is an open-access article distributed under the terms of the Creative Commons Attribution License (CC BY). The use, distribution or reproduction in other forums is permitted, provided the original author(s) or licensor are credited and that the original publication in this journal is cited, in accordance with accepted academic practice. No use, distribution or reproduction is permitted which does not comply with these terms. 